\title{
Macula Erythematous Rash Due to Temozolomide Involving Head and Neck Region Only
}

\author{
Sunil Shrestha ${ }^{{ }^{*}}$, Sudip Shrestha ${ }^{2}$, Bijesh Raj Ghimire ${ }^{2}$, Sachin Shakya ${ }^{2}$ Gisupnikha Prasiko ${ }^{3}$ \\ and Binaya Sapkota ${ }^{4}$ \\ ${ }^{1}$ Department of Clinical Pharmacy, Nepal Cancer Hospital and Research Centre, Harisiddhi, Lalitpur, Nepal \\ ${ }^{2}$ Department of Medical Oncology, Nepal Cancer Hospital and Research Center, Harisiddhi, Lalitpur, Nepal \\ ${ }^{3}$ Department of Radiation Oncology, Nepal Cancer Hospital and Research Center, Harisiddhi, Lalitpur, Nepal \\ ${ }^{4}$ Faculty of Health Science, Department of Pharmaceutical Sciences, Nobel College, Sinamangal, Kathmandu, Pokhara \\ University, Nepal
}

Received: February 02, 2018; Accepted: March 05, 2018; Published: March 09, 2018

*Corresponding author: Sunil Shrestha, Clinical Pharmacist and Research Associate, Department of Clinical Pharmacy, Nepal Cancer Hospital and Research Center Pvt. Ltd, Harisiddhi, Lalitpur, Nepal, E-mail: sunilcresta@gmail.com

\begin{abstract}
The main aim of the presentstudywas to investigate a case of macula erythematous rash involving head and neck region only associated with the use of temozolomide in the treatment of glioblastomamultiformebrain tumor-grade IV-WHO. In the present case study, we presented a case of a patient with glioblastomamultiforme-brain tumor-grade IV-WHO. Patient after surgery was followed by radiotherapy and chemotherapy, and after cycles of chemotherapy with temozolomide came with the case of macula erythematous rash.
\end{abstract}

\section{Background}

In adults, glioblastoma is the most well-known forceful essential Central Nervous System (CNS) tumor which represents $45.6 \%$ of all harmful CNS tumors, which has an occurrence of 3.19 for every 100,000 [1]. For the treatment of glioblastoma, the current level of treatment is surgical resection took after by adjuvant temozolomide and accompanying radiotherapy. The expansion of temozolomide to radiotherapy essentially enhances general mediansurvival and progression free survival contrasted with radiotherapy alone. Temozolomide is an alkylating agent and is, for the most part, an all-around endured treatment with exhaustion, thromboembolic occasions, and lymphopenia as the most widely recognized side effects. Grade III or IV myelosuppression is a generally uncommon symptom announced in $4 \%$ of patients [2].

We reported a probable case of macula erythematousrash due to temozolomide involving head and neck region only. Causality and seriousness of the response were evaluated utilizing the Naranjo and Hartwig scales, individually $[3,4]$.

\section{Case Description}

A case of 55-year-old female patient, who was apparently well one month back. Then she developed headache, insidious in onset, generalized on and off type, throbbing type of headache, no any relieving and aggravating factors, and also complained of decreased appetite. There was no history of fever, nausea, vomiting, loss of consciousness, and abnormal body movement.

She had history of decompressive surgery for type I chiraimalformation on 12/12/2013. She also had history of postoperative meningitis, which was managed conservatively. There was no any history of medical illness in the past. Histopathological features were consistent with glioblastomamultiforme (WHO Grade IV).

Patient was admitted for further evaluation and management. She had a severe refractory headache when she underwent investigations. She had left temporal high-grade lesion and operation was done on 21/05/2017. Post-operatively, she was managed with IV antibiotics, intravenous fluids, proton pump inhibitors (Pantoprazole 40mg), analgesics and inj. dexamethasone.

After surgery, she was referred to Nepal Cancer Hospital and Research Centre and started with chemotherapy before starting radiotherapy during Coordinated Reset simulation. Patient after surgery was followed by radiotherapy and chemotherapy. After cycles of chemotherapy with temozolomide she was on oral capsule temozolomide $250 \mathrm{mg}$.

On 17/01/2018, she visited the clinic complaining dry mouth, erythema, and mucositis from $03 / 01 / 2018$. On casualty assessment, we assessed it as "probable" due to temozolomide. On observation, it was diagnosed as macula erythematous rash involving head and neck region which was painful and with the suspected vasculitis. She was prescribed with Prednisolone 20 mg, Cloben G (Combination of Clotrimazole, Beclomethasone, Gentamycin) which has an antibacterial, Antifungal and antiallergic action cream and Antiseptic pain relieving gel (Gel containing Choline Salicylate and Benzalkonium Chloride Solution) was given. After a follow-up after a week i.e. on $24 / 01 / 2018$, patient's sign and symptoms was resolved with the prescribed medicines. 


\section{Discussion}

The detailed dermatologic reactions of temozolomide are deficient however it comprises of urticaria, desquamative and maculopapular rash, alopecia, Stevens-Johnson disorder/harmful epidermal necrolysis, and palmoplantar erythrodysesthesia [5]. Pothiawala et al. supported that the apparent little occurrence of cutaneous unfriendly medication responses (ADRs) of temozolomide may be halfway clarified by the parallel utilization of corticosteroids, which are more than once utilized as a part of patients with high-review gliomas to diminish the edema related with radiation or tumor development [6]. The incidence of Macula erythematousrash in general population is unknown. The data related to this incidence was unavailable.

For a foundation of a causal connection between a medication and suspected ADRs, Causality Assessment is a widely utilized framework. The Naranjo Algorithm is regularly used to assess the causality of ADRs and depends on a score ascertained from the responses to 10 questions. On a scale with a most extreme of "13" focuses, a score more prominent than "9" affirms that the unfavorable response to the medication is related with the presume drug. A score in the vicinity of " 5 and 8 " is viewed as "Probable", while a score of " 1 to 4 " is delegated "Possible" [3]. For our situation, the causality appraisal uncovered that the ADR was "plausible". Evaluation of the seriousness of ADRs can give valuable data and guide activities towards dealing with these. The Hartwig scale arranges ADRs as "mild", "moderate" or "severe" [4]. For our situation in presenting case, the presumed drug i.e., temozolomide was proceeded with; along these lines, fitting the 'mild' classification. Medicinal Oncologists ought to stay mindful of the way that antagonistic skin responses hold on in exorbitant screening. It is vital to know the possibly dangerous harmfulness of each chemotherapeutic operator, including temozolomide. Temozolomide ought to be endorsed with the acknowledgment of conceivable reactions announced here.

\section{Limitations of the Study}

Since the present case report was a solitary instance of its write, all the more vast scale looks into are justified to investigate and clarify points of interest of the certainties of macula erythematous rash possibly caused by temozolomide.

\section{Declarations}

Ethical approval and consent to participate: Patient consent was taken from the patient's party.

\section{References}

1. Ostrom QT, Gittleman H, Liao P, Rouse C, Chen Y, Dowling J, et al. CBTRUS statistical report: primary brain and central nervous system tumors diagnosed in the United States in 2007-2011. Neuro Oncol. 2014;16 Suppl 4:iv1-iv63. doi: 10.1093/neuonc/nou223

2. Stupp R, Mason WP, van den Bent MJ, Weller M, Fisher B, Taphoorn MJ, et al. Radiotherapy plus concomitant and adjuvant temozolomide for glioblastoma. N Engl J Med. 2005;352(10):987-996.

3. Naranjo CA, Busto U, Sellers EM, Sandor P, Ruiz I, Roberts EA, et al. A method for estimating the probability of adverse drug reactions. Clin Pharmacol Ther. 1981;30(2):239-245.

4. Hartwig SC, Siegel J, Schneider PJ. Preventability and severity assessment in reporting adverse drug reactions. Am J Hosp Pharm. 1992;49(9):2229-2232.

5. Alonso-Llamazares A, Vega-Castro A, Beitia-Mazuecos JM, MateoBorrega B, Cardenas-Contreras R. Rapid desensitization with temozolomide in patients with delayed maculopapular rash. J Investig AllergolClin Immunol. 2012;22(6):448-449.

6. Pothiawala S, Hsu MY, Yang C, Kesari S, Ibrahimi OA. Urticarial hypersensitivity reaction caused by temozolomide. J Drugs Dermatol. 2010;9(9):1142-1144. 